\section{Volume 60}

Issue 60, I (pag. 3- 96): 14-7-1978

Issue 60, 2 (pag. 99-196): 18-8-1978

Issue 60, 3 (pag. 199-280): 8-9-1978

\title{
INDEX AUCTORUM
}

Armitage, P. D., 229

Bakker, C., 93

Birkholm-Hansen, S., 235

Biswas, S., 2 I 3

Bond, W. J., 243

Borghouts, C. H., 193

Bott, T. L., 3

Bottom, D., 203

Brock, J. T., 3

Casterlin, M. E., 89

Cattaneo, A., I 35

Cushing, C. E., 3

Czeczuga, B., I73

Deshpande, U. D., I 25

Drescher, Tn. G. N. 279

El-Shamy, F. M., I I 3

Empain, A., 49

Flowers, R. W., 159

Goel, P. K., I 87

Gopal, B., I 87

Gophen, M., I 7

Gregory, S. V., 3

Guha, G., 75

Hansen, F. S., 235
Hanumante, M. M., I 25

Hilsenhoff, W. L., I 59

Howell, L. W., I 29

Hynes, H. B. N., I 77, 275

Janković, M. J., $26 \mathrm{I}$

Jones, R. K. H., 8 I

Kalff, J., I 35

King, D., 3

Key, A. J., 195

Klyszejko, B., I 73

Krutchkoff, R. G., I 29

Latey, A. N., 22 I

Lush, D. L., I77, 27 I

Maeseneer, J. de I 5 I

Maurer, D., 203

Mendl, H., I09

Moeller, R. E., 13

Mokrey, J. E., 213

Moss, B., 23

Moth Iversen, T., 235

Nagabhushanam, R., I 25

Olofsson, S., 99

Parker, B. C., I 29

Pauw, M. de I5I
Pembroke, A., 203

Petersen, R. C., 3

Rangneker, P. V., 221

Reynolds, W. W., 89

Roberts, M. G., 259

Roos, P. J., I 96

Roskoski, J. P., I 3

Russo, A. R., 33

Sharma, B. K., I9I

Sharma, K. P., I 87

Sharma, S. N., 75

Singh, B. R., 75

Soest, R. W. M. v., 278

Spoel, S. v. d., I 93

Tol, J. v., 278

Trivedy, R. K., I 87

Utrecht, W. L. v., 278

Waegeman, D., I 5 I

Watling, L., 203

Wiberg-Larsen, P., 235

Wilde, P. A. W. J. de 194

Yadav, A. N., 75

Yocum, W. L., I99

Young, J. O., I 45 